\title{
Searching for Minimum in Dependence of Squared Speed-of-Sound on Collision Energy
}

\author{
Fu-Hu Liu, ${ }^{1}$ Li-Na Gao, ${ }^{1}$ and Roy A. Lacey ${ }^{2}$ \\ ${ }^{1}$ Institute of Theoretical Physics, Shanxi University, Taiyuan, Shanxi 030006, China \\ ${ }^{2}$ Departments of Chemistry \& Physics, Stony Brook University, Stony Brook, NY 11794, USA \\ Correspondence should be addressed to Fu-Hu Liu; fuhuliu@163.com
}

Received 25 March 2016; Accepted 21 April 2016

Academic Editor: Ming Liu

Copyright (c) $2016 \mathrm{Fu}-\mathrm{Hu}$ Liu et al. This is an open access article distributed under the Creative Commons Attribution License, which permits unrestricted use, distribution, and reproduction in any medium, provided the original work is properly cited. The publication of this article was funded by SCOAP . $^{3}$

\begin{abstract}
Experimental results of the rapidity distributions of negatively charged pions produced in proton-proton $(p-p)$ and berylliumberyllium $(\mathrm{Be}-\mathrm{Be})$ collisions at different beam momentums, measured by the NA61/SHINE Collaboration at the super proton synchrotron (SPS), are described by a revised (three-source) Landau hydrodynamic model. The squared speed-of-sound parameter $c_{s}^{2}$ is then extracted from the width of rapidity distribution. There is a local minimum (knee point) which indicates a softest point in the equation of state (EoS) appearing at about $40 \mathrm{~A} \mathrm{GeV} / c$ (or $8.8 \mathrm{GeV}$ ) in $c_{s}^{2}$ excitation function (the dependence of $c_{s}^{2}$ on incident beam momentum (or center-of-mass energy)). This knee point should be related to the searching for the onset of quark deconfinement and the critical point of quark-gluon plasma (QGP) phase transition.
\end{abstract}

\section{Introduction}

Comparing with the relativistic heavy ion collider (RHIC) in USA [1-3] and the large hadron collider (LHC) in Switzerland $[4,5]$, the fixed target experiments performed at the super proton synchrotron (SPS) in Switzerland [6,7] present relatively simple and clean collisions process. The multiplicity in collisions at the SPS is also low comparing with those at the RHIC and LHC. As one of the "first day" measurement quantities, the rapidity (pseudorapidity) distributions of charged particles are reported by experimental collaborations [1-7]. These distributions give us a chance to analyze longitudinal picture of particle productions. At the same time, based on the rapidity distributions, one can extract other information such as the penetrating (stopping) power of projectile (target) nucleus, energy and rapidity losses of projectile nucleus, and energy and particle densities of interacting region.

As a measurement of particle density and mean free path, the squared speed-of-sound parameter which characterizes partly the formation of matters in interacting region can be extracted from the width of Gaussian rapidity distribution described by the Landau hydrodynamic model [8-18].
Generally, the rapidity spectra obtained in collisions at the SPS, RHIC, and LHC are not simply Gaussian distributions. Instead, two-Gaussian distributions, one in the backward (target) region and the other in the forward (projectile) region, are needed to fit the experimental data $[6,19,20]$. However, the two-Gaussian distribution implies that there is no central source at midrapidity, which seems unbelievable. According to the three-source relativistic diffusion model [21-24], a central source arising from interactions between low-momentum gluons in both target and projectile should be located at midrapidity, a target-like (projectile-like) source arising from interactions between valence quarks in the target (projectile) and low-momentum gluons in the projectile (target) are expected to appear in the backward (forward) rapidity region.

To extract the squared speed-of-sound parameter so that the formation of matters can be characterized by an alternative method, we need the Landau hydrodynamic model [8-10] and its improved or simplified version [11-18] in the descriptions of rapidity distributions. In fact, the Landau hydrodynamic model is not good enough to fit the whole rapidity range. In our recent work $[25,26]$, we have revised 
the Landau hydrodynamic model [8-18] to a three-source situation. The rapidity distributions measured in experiments are then described by three Gaussian distributions. This picture is consistent with the three-source relativistic diffusion model [21-24].

In this paper, we use the revised (three-source) Landau hydrodynamic model $[25,26]$ to describe the rapidity distributions of negatively charged pions produced in protonproton $(p-p)$ and beryllium-beryllium (Be-Be, exactly ${ }^{7} \mathrm{Be}-$ ${ }^{9} \mathrm{Be}$ ) collisions at different beam momentums measured by the NA61/SHINE Collaboration at the SPS. Combining with our previous works $[25,26]$, we observe the dependence of squared speed-of-sound on collision energy in a wide range.

The rest of this paper is structured as follows. The model is shortly described in Section 2. Results and discussion are given in Section 3. In Section 4, we summarize our main observations and conclusions.

\section{The Model}

The revised (three-source) Landau hydrodynamic model used in the present work can be found in our previous work $[25,26]$. To give a short and clear description of the model, we introduce the main results of the model in the following.

The Landau hydrodynamic model [8-18] results approximately in a Gaussian rapidity distribution which does not exactly describe the experimental data $[6,19,20]$. We have revised the model to three sources: a central source which locates at midrapidity and covers the rapidity range as wide as possible, and a target (projectile) source which locates in the backward (forward) region and revises the rapidity distribution from the central source $[25,26]$. The experimental rapidity distributions are then described by three Gaussian functions. And based on the three Gaussian functions, the experimental pseudorapidity distributions can be described by a method which makes a distinction between rapidity and pseudorapidity.

According to the Landau hydrodynamic model [8-18] and our revision $[25,26]$, the rapidity distribution, $d N_{\mathrm{ch}} / d y$, of charged particles produced in a given source in high energy collisions can be described by a Gaussian function:

$$
\frac{d N_{\mathrm{ch}}}{d y}=\frac{N_{0}}{\sqrt{2 \pi} \sigma_{X}} \exp \left[-\frac{\left(y-y_{X}\right)^{2}}{2 \sigma_{X}^{2}}\right] \text {, }
$$

where $\sigma_{X}, y_{X}$, and $N_{0}$ denote the width, peak position, and normalization constant, respectively; $X=C, T$, and $P$ are for the central, target, and projectile sources, respectively. The experimental result is in fact a sum weighted by the three Gaussian functions. $c_{s}^{2}(\mathrm{X})$ is

The relation between $\sigma_{X}$ and the squared speed-of-sound

$$
\sigma_{X}=\sqrt{\frac{8}{3} \frac{c_{s}^{2}(X)}{1-c_{s}^{4}(X)} L}
$$

where $L=\ln \left(\sqrt{s_{N N}} / 2 m_{p}\right)$ is the logarithmic Lorentz contraction factor which is independent of $X, \sqrt{s_{N N}}$ denotes the center-of-mass energy per pair of nucleons in nucleusnucleus collisions and is simplified to $\sqrt{s}$ in $p-p$ collisions, and $m_{p}$ denotes the rest mass of a proton. From $(2), c_{s}^{2}(X)$ is expressed by using $\sigma_{X}$ to be

$$
c_{s}^{2}(X)=\frac{1}{3 \sigma_{X}^{2}}\left(\sqrt{16 L^{2}+9 \sigma_{X}^{4}}-4 L\right) .
$$

In the above extractions of $\sigma_{X}$ and $c_{s}^{2}(X)$ from the rapidity distribution, we distinguish accurately the rapidity and pseudorapidity distributions $[25,26]$. In the case of representing the pseudorapidity distribution in experiment, we also extract $\sigma_{X}$ and $c_{s}^{2}(X)$ from the hidden rapidity distribution. Our treatment ensures the method of extraction being concordant.

\section{Results and Discussion}

Figure 1 presents the rapidity distributions, $d n / d y$, of negatively charged pions produced in $p$ - $p$ and ${ }^{7} \mathrm{Be}-{ }^{9} \mathrm{Be}$ collisions at the SPS. For $p$ - $p$ collisions, the incident momentums for Figures 1(a)-1(e) are 20,31, 40, 80, and $158 \mathrm{GeV} / c$, respectively, which correspond to $\sqrt{s}=6.3,7.7,8.8$, 12.3, and $17.3 \mathrm{GeV}$, respectively. For ${ }^{7} \mathrm{Be}-{ }^{9} \mathrm{Be}$ collisions, the incident momentums for Figures 1 (a)-1(e) are 20A, 31 A, $40 A, 75 A$, and $150 A \mathrm{GeV} / c$, respectively, which correspond to $\sqrt{s_{N N}}=6.3,7.7,8.8,11.9$, and $16.8 \mathrm{GeV}$, respectively. The symbols represent the experimental data of the NA61/SHINE Collaboration $[6,7]$ and the curves are our results fitted by the revised (three-source) Landau hydrodynamic model. Different centrality classes $(0-5 \%, 5-10 \%, 10-15 \%$, and $15-$ $20 \%$ ) for ${ }^{7} \mathrm{Be}-{ }^{9}$ Be collisions are presented by different symbols marked in the panels. The values of fit parameters, $\sigma_{C}, \sigma_{T}$ $\left(=\sigma_{P}\right), y_{C}$, rapidity shift $\Delta y\left(=y_{C}-y_{T}=y_{P}-y_{C}\right)$, relative contribution $k_{T}$ of the target source $\left(=k_{P}\right.$, the relative contribution of the projectile source), and normalization constant $N_{0}$, are listed in Table 1 with the values of $\chi^{2}$ per degree of freedom (dof). The last two columns in Table 1 present the values of $c_{s}^{2}(C)$ and $c_{s}^{2}(T)\left[=c_{s}^{2}(P)\right]$, which are calculated from (3) due to $\sigma_{X}$. Both $c_{s}^{2}(C)$ and $c_{s}^{2}(T)\left[=c_{s}^{2}(P)\right]$ are in the units of $c^{2}$, where $c$ is the speed of light in vacuum. One can see that the model describes approximately the experimental data in most cases.

To see clearly the relation between parameter and energy, Figures 2(a) and 2(b) give the dependences of main free parameter $\sigma_{X}$ and extracted parameter $c_{s}^{2}(X)$ on $\sqrt{s_{N N}}$, respectively. The results corresponding to $p$ - $p$ collisions and ${ }^{7} \mathrm{Be}-{ }^{9} \mathrm{Be}$ collisions with different centrality classes are displayed by different symbols, which reflect a part of parameter values listed in Table 1 . The line segments for $p$ - $p$ collisions and for ${ }^{7} \mathrm{Be}-{ }^{9} \mathrm{Be}$ collisions with centrality class $15-20 \%$ are given for guiding the eyes. One can see that $\sigma_{C}$ increases with increase of $\sqrt{s_{N N}}, \sigma_{T}\left(\sigma_{P}\right)$ does not show a change with $\sqrt{s_{N N}}, c_{s}^{2}(C)$ shows itself a local minimum (knee point) which indicates a softest point in the equation of state (EoS) at $\sqrt{s_{N N}}=8.8 \mathrm{GeV}$, and $c_{s}^{2}(T)\left[c_{s}^{2}(P)\right]$ decreases with $\sqrt{s_{N N}}$. The dependences of other parameters $\left(y_{C}, \Delta y, k_{T}\right.$, and $\left.N_{0}\right)$ on energy are not analyzed due to trivialness. 


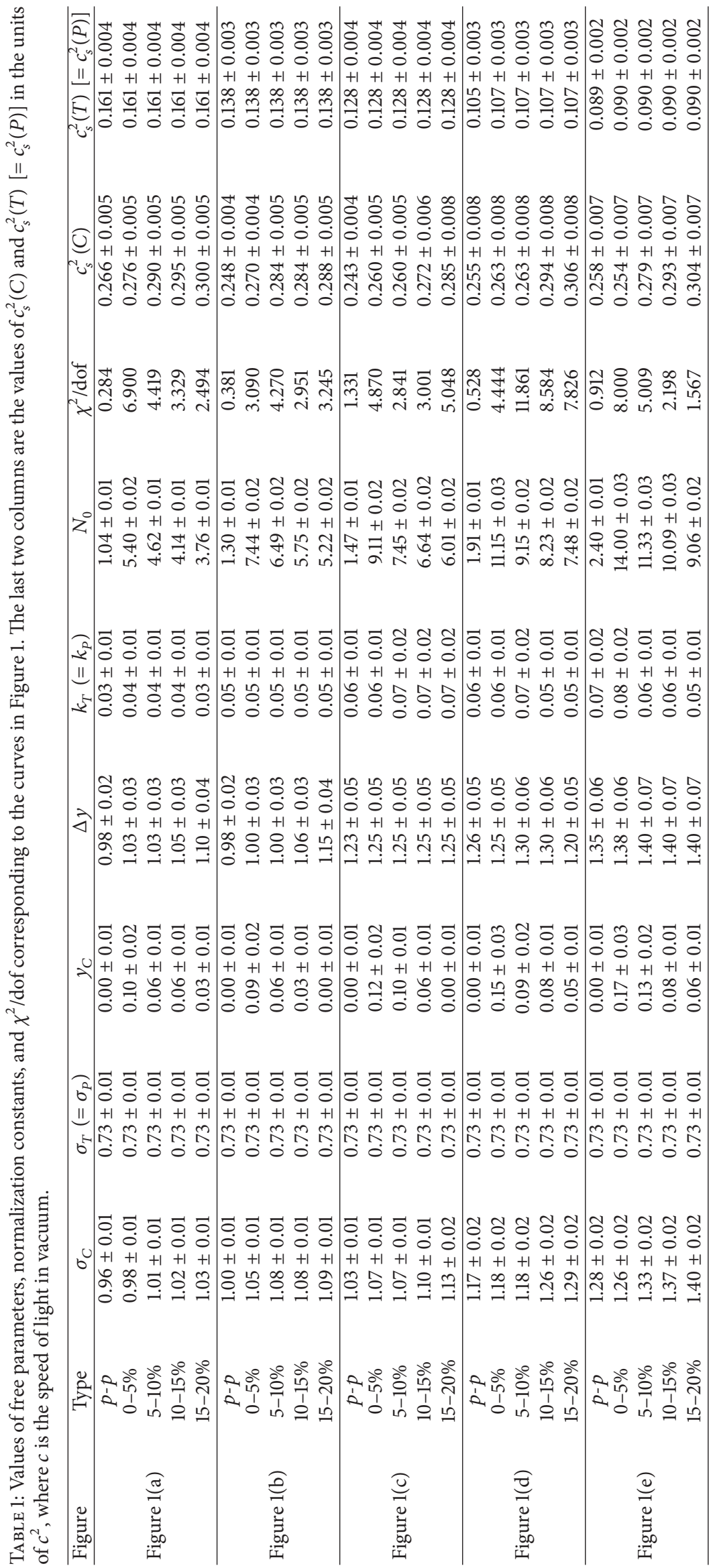




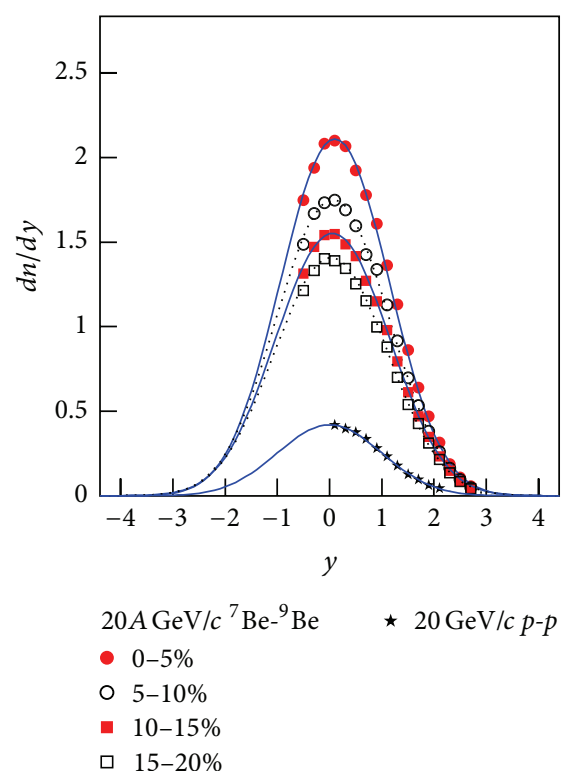

(a)

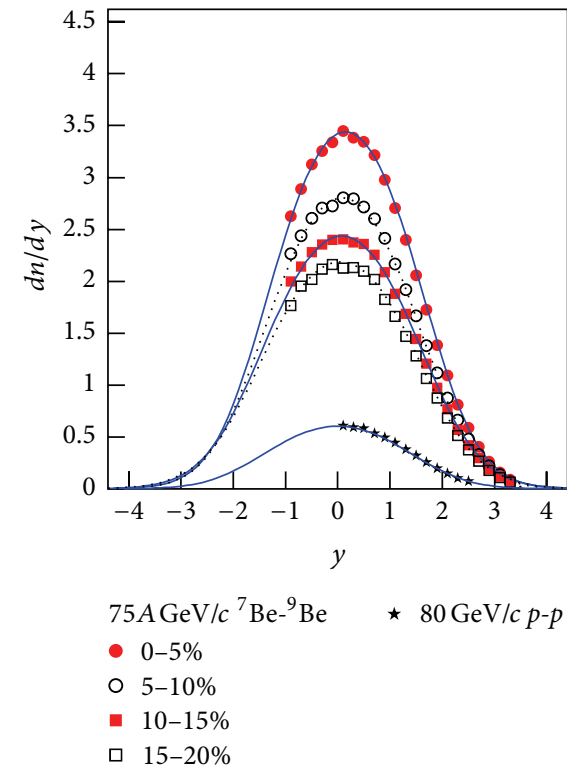

(d)

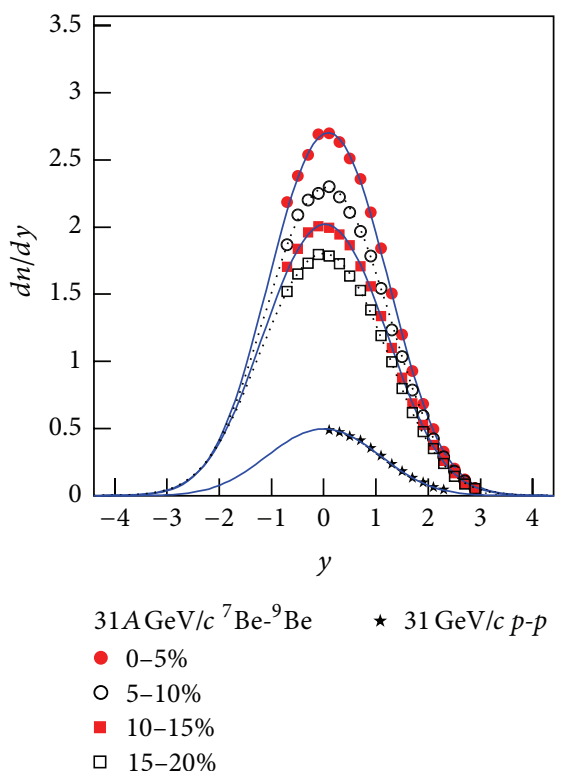

(b)

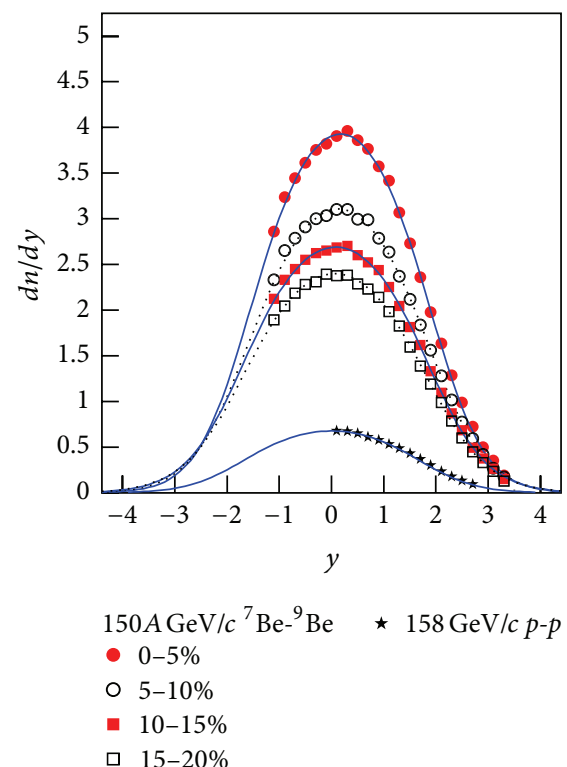

(e)

Figure 1: Rapidity distributions of negatively charged pions produced in $p-p$ and ${ }^{7} \mathrm{Be}-{ }^{9} \mathrm{Be}$ collisions at the SPS. (a)-(e) Corresponding to different beam momentums marked in the panels. The symbols represent the experimental data of the NA61/SHINE Collaboration [6,7] and the curves are our results fitted by the revised (three-source) Landau hydrodynamic model.

To study the knee point in detail, Figures 3(a) and 3(b) show $\sigma_{C}$ and $c_{s}^{2}(C)$ excitation functions (the dependences of $\sigma_{C}$ and $c_{s}^{2}(C)$ on $\left.\sqrt{s_{N N}}\right)$, respectively, where the results for $p$ - $p$ and Be-Be collisions at lower energies $(\leq 17.3 \mathrm{GeV})$ are taken from Figure 2 (Table 1) and the results for $p$ $p$, proton-antiproton $(p-\bar{p})$, copper-copper $(\mathrm{Cu}-\mathrm{Cu})$, goldgold $(\mathrm{Au}-\mathrm{Au})$, and lead-lead $(\mathrm{Pb}-\mathrm{Pb})$ collisions at higher energies $(\geq 19.6 \mathrm{GeV})$ are taken from our previous works $[25,26]$. Different symbols represent different collisions, which are marked in the panels. The symbols with large size denote central collisions, and the symbols with small

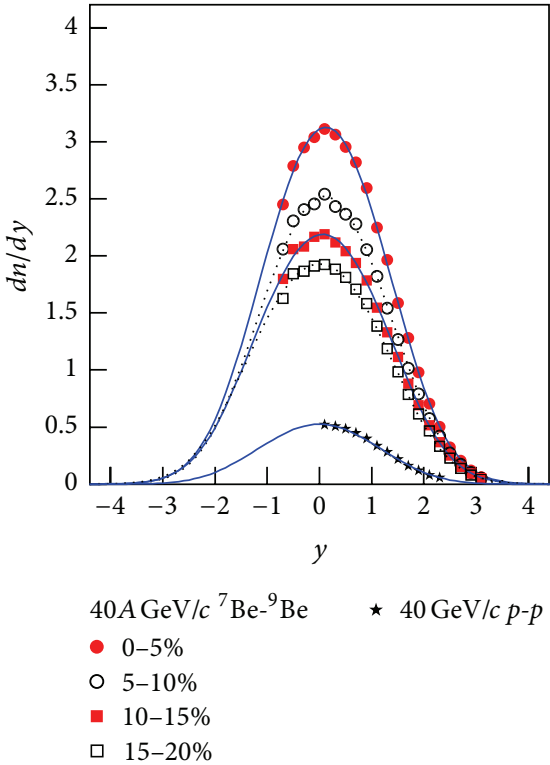

(c) size denote noncentral collisions. The centrality classes for $\mathrm{Cu}-\mathrm{Cu}$ collisions are from $0-3 \%$ to $50-55 \%$ [27]; for $\mathrm{Au}-$ $\mathrm{Au}$ collisions from $0-3 \%$ to $45-50 \%$ (maximum $40-45 \%$ at $19.6 \mathrm{GeV}$ ) [27]; and for $\mathrm{Pb}-\mathrm{Pb}$ collisions from $0-5 \%$ to $20-30 \%$ [28]. The line segments for $p$ - $p$ collisions at SPS and higher energies are given for guiding the eyes, and the two dotted lines in Figure 3(b) show $c_{s}^{2}(C)=1 / 3$ and $1 / 2$, respectively. One can see that at higher energies $\sigma_{C}$ increases with increase of $\sqrt{s_{N N}}$ and most $c_{s}^{2}(C)$ are between $1 / 3$ and $1 / 2$ [a few $c_{s}^{2}(C)$ are beyond $1 / 2$ due to statistical fluctuations]. 


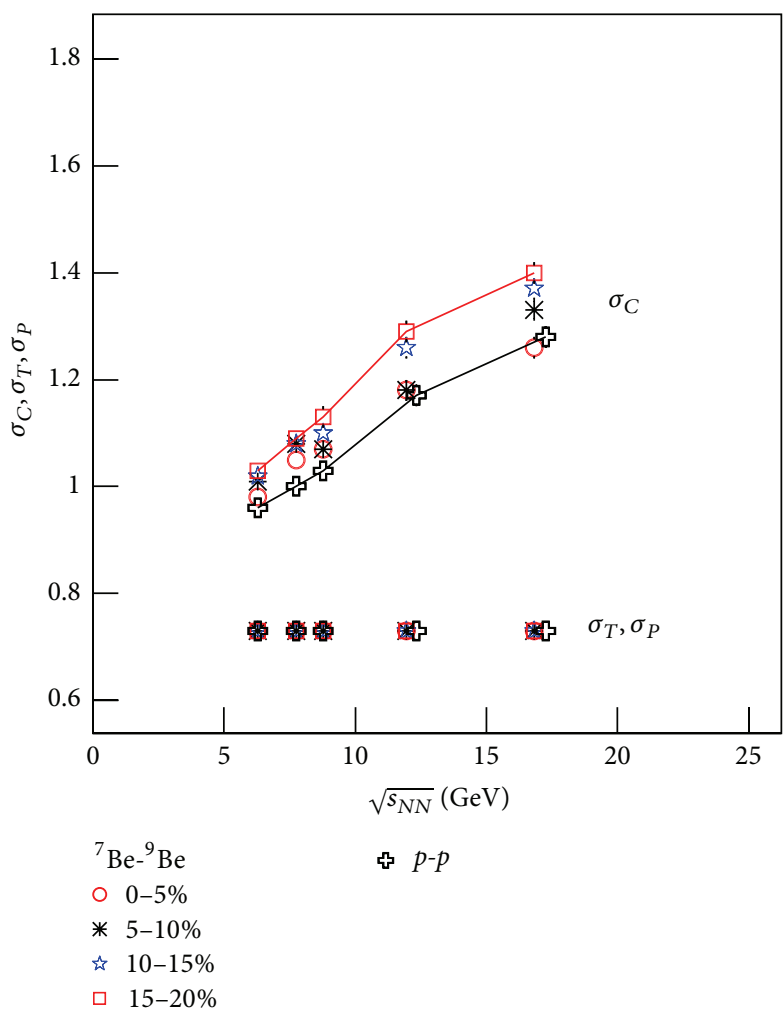

(a)

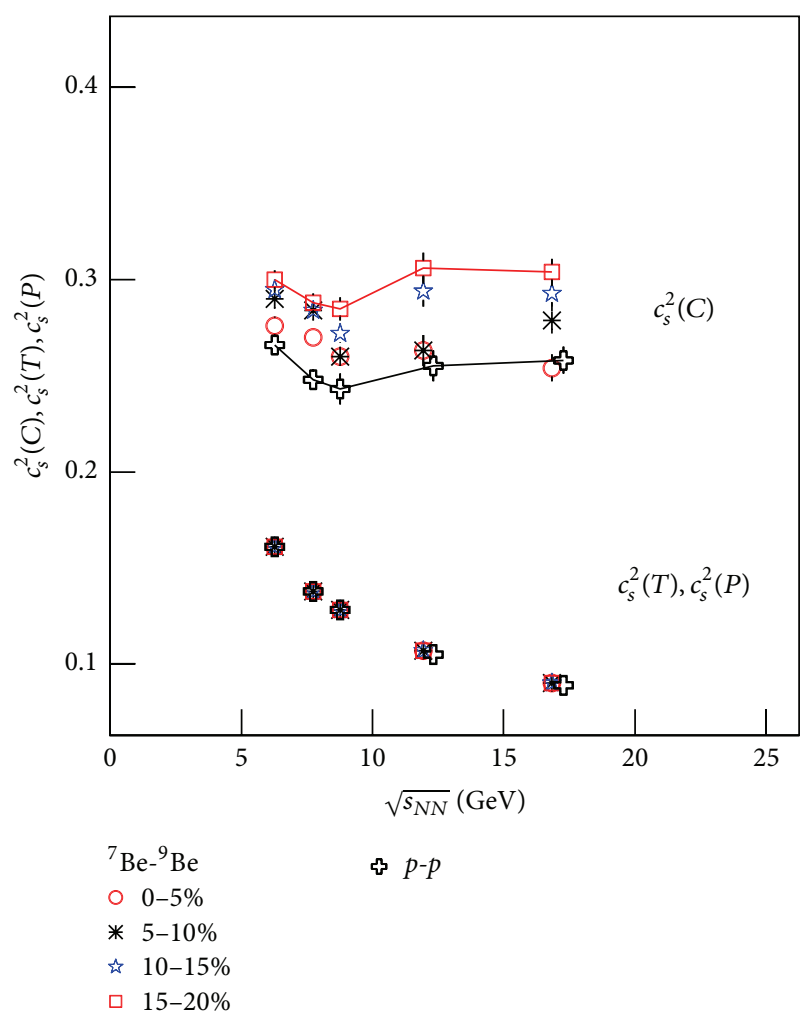

(b)

Figure 2: Dependences of (a) $\sigma_{X}$ and (b) $c_{s}^{2}(X)$ on $\sqrt{s_{N N}}$. The symbols represent the values listed in Table 1 and the line segments for two cases are given for guiding the eyes. Different symbols correspond to different types (centralities) of collisions.

In particular, one can see some detailed laws in $c_{s}^{2}(C)$ excitation function (the dependence of $c_{s}^{2}(C)$ on $\sqrt{s_{N N}}$ ). (i) In the lower energy range, $c_{s}^{2}(C)$ decreases with increase of $\sqrt{s_{N N}}$, then the first knee point appears at $\sqrt{s_{N N}}=8.8 \mathrm{GeV}$, and afterwards $c_{s}^{2}(C)$ increases with increase of $\sqrt{s_{N N}}$. (ii) From 17.3 to $19.6 \mathrm{GeV}, c_{s}^{2}(C)$ has a jump from $\leq 0.3$ to $1 / 3-$ $1 / 2$. (iii) In the higher energy range, $c_{s}^{2}(C)$ seems not related to $\sqrt{s_{N N}}$, and a saturation appears. The first knee point is a local minimum which indicates a softest point in the EoS. As the predecessor of $c_{s}^{2}(C), \sigma_{C}$ increases always with increase of $\sqrt{s_{N N}}$. We do not observe a softest point in the EoS in $\sigma_{C}$ excitation function. These abundant phenomena should be related to the searching for the onset of deconfinement of the quarks and gluons in proton-proton collisions and the critical point of phase transition from hadronic matter to quark-gluon plasma (QGP) in nucleus-nucleus collisions.

The softest point $(8.8 \mathrm{GeV})$ obtained in the present work is compatible with the previous works $[29,30]$ which used the Landau hydrodynamic model and the ultra-relativistic quantum molecular dynamics hybrid approach and indicated the softest point locating in the energy range from 4 to $9 \mathrm{GeV}$. Other works which study dependences of ratio of numbers of positive kaons and pions $\left(K^{+} / \pi^{+}\right)[20,31,32]$, chemical freeze-out temperature $\left(T_{\mathrm{ch}}\right)[31,32]$, mean transverse mass minus rest mass $\left(\left\langle m_{T}\right\rangle-m_{0}\right)$ [31], and ratio of widths of experimental negative pion rapidity distribution and Landau hydrodynamic model prediction $\left[\sigma_{y}\left(\pi^{-}\right) / \sigma_{y}\right.$ (hydro)] [32] on $\sqrt{s_{N N}}$ show knee point around $7-8 \mathrm{GeV}$. A wiggle in the excitation function of a specific reduced curvature of the netproton rapidity distribution at midrapidity is expected in the energy range from 4 to $8 \mathrm{GeV}[33,34]$. However, the searching for the onset of quark deconfinement and the critical point of QGP phase transition is a complex process [35]. The relation between the softest point and onset of deconfinement is still an open question. We need to analyze more other experimental data so that these results can be confirmed each other.

As for the jump of $c_{s}^{2}(C)$ from $\leq 0.3$ to $1 / 3-1 / 2$ when $\sqrt{s_{N N}}$ increases from 17.3 to $19.6 \mathrm{GeV}$, we explain it as the result of changing the mean free path of produced particles, if the statistical fluctuation is excluded. At the SPS energies, the interacting system has a small density due to low collision energy and the produced particles have a large mean free path which results from a gas-like state and results in a small $c_{s}^{2}$. The situation at the RHIC or LHC energies is opposite, where the interacting system has a large density due to high collision energy and the produced particles have a small mean free path which results from a liquid-like state and results in a large $c_{s}^{2}$. Let $D$ denote the dimensionality of space. According to Refs. [36, 37], we have the relation of $c_{s}^{2}=1 / D$ for massless particles. The particles at the gas-like state have a larger probability to appear in three-dimensional space, which results in the maximum $c_{s}^{2}$ being $1 / 3$ which is the 


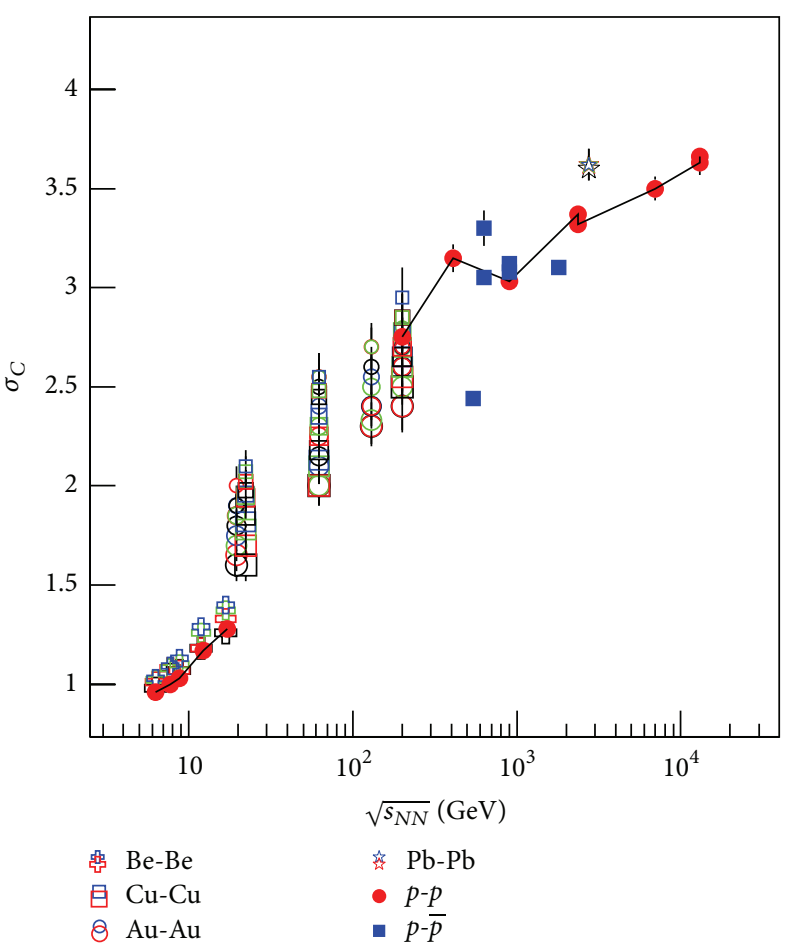

(a)

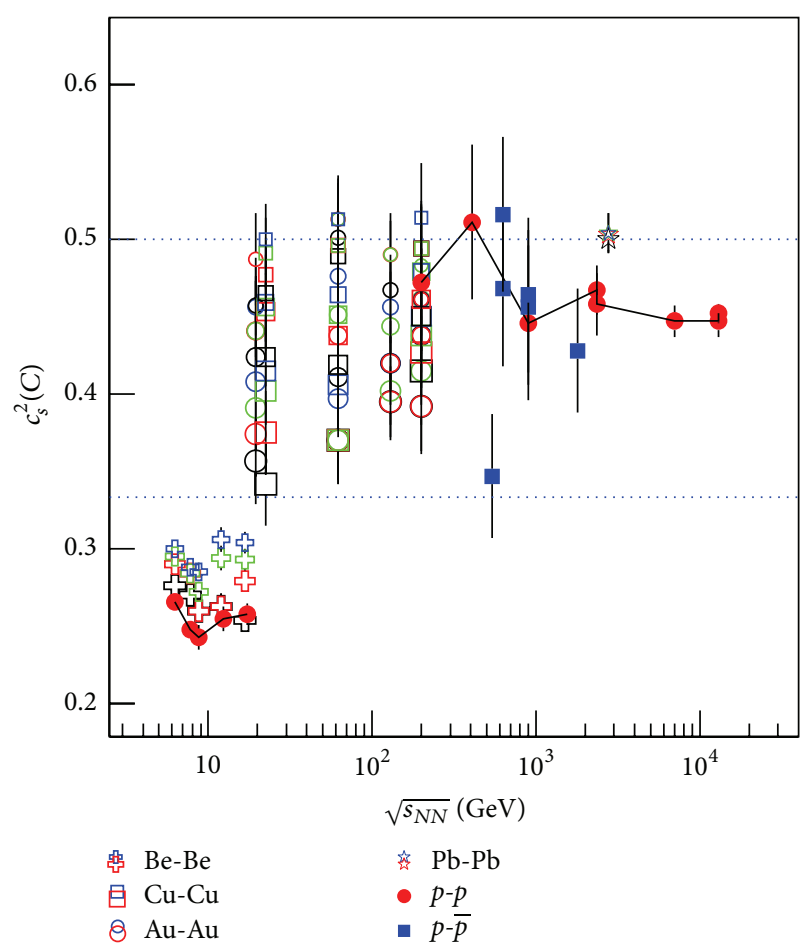

(b)

FIGURE 3: Dependences of (a) $\sigma_{C}$ and (b) $c_{s}^{2}(C)$ on $\sqrt{s_{N N}}$. The symbols represent the values listed in Table 1 (for the energy range $\leq 17.3 \mathrm{GeV}$ ), or taken from our previous work $[25,26]$ (for the energy range $\geq 19.6 \mathrm{GeV}$ ). The line segments for $p$ - $p$ collisions at SPS and higher energies are given for guiding the eyes, and the two dotted lines in $(\mathrm{b})$ show $c_{s}^{2}(C)=1 / 3$ and $1 / 2$, respectively. We would like to point out that we distinguish different objects by shapes not colors. The black and green objects have the same meanings as those of red and blue ones.

situation at the SPS energies. The particles at the liquid-like state have a larger probability to appear in two-dimensional space, which results in the maximum $c_{s}^{2}$ being $1 / 2$ which is the situation at the RHIC and LHC energies.

To see the relations of $\sigma_{C}$ and other parameters at the SPS energies which show the softest point in the EoS, Figures 4(a), $4(\mathrm{~b}), 4(\mathrm{c})$, and $4(\mathrm{~d})$ present the dependences of $\sigma_{C}$ on $y_{C}$, $\Delta y, k_{T}$, and $N_{0}$, respectively, where the closed circles, open circles, closed squares, open squares, and stars correspond to the collision energy being 6.3, 7.7, 8.8, 12.3 (11.9), and 17.3 (16.8) $\mathrm{GeV}$, respectively, which are taken from Table 1. One can see that $\sigma_{C}$ does not show an obvious dependence on $y_{C}$ and $k_{T}$, though $k_{T}$ shows somehow a saturation. $\sigma_{C}$ increases with increases of $\Delta y$ and $N_{0}$ due to the latter two increasing with increase of $\sqrt{s_{N N}}$. The relation between $\sigma_{C}$ and $\sqrt{s_{N N}}$ is the most important one among all the relations. Other relations such as the relations between $\sigma_{C}$ and $y_{C}, \Delta y, k_{T}$, as well as $N_{0}$ are less important. To present the relations between $c_{s}^{2}(C)$ and $y_{C}, \Delta y, k_{T}$, as well as $N_{0}$ is trivial due to $c_{s}^{2}(C)$ being calculated from $\sigma_{C}$.

To study the most important relation between $\sigma_{C}$ and $\sqrt{s_{N N}}$ in detail, Figure 5(a) displays a few examples of linear relations between $\sigma_{C}$ and $\ln \sqrt{s_{N N}}$ which reflect approximately the main area of parameter points in Figure 3(a). The solid lines corresponded to cases A, B, C, and D and can be expressed by $\sigma_{C}=0.5 \ln \sqrt{s_{N N}}+0.3, \sigma_{C}=0.3 \ln \sqrt{s_{N N}}+$ $0.5, \sigma_{C}=0.2 \ln \sqrt{s_{N N}}+0.6$, and $\sigma_{C}=0.1 \ln \sqrt{s_{N N}}+0.7$, respectively. After conversion by (3), the four similar lines of $\sigma_{C}-\ln \sqrt{s_{N N}}$ show very different relations of $c_{s}^{2}(C)-$ $\ln \sqrt{s_{N N}}$ given correspondingly in Figure 5(b). Cases A and B show obvious minimums, while cases $C$ and D do not show any minimum. Decreasing the intercept in case $\mathrm{A}$ from 0.3 to 0.2 and 0.1 , respectively, the corresponding results presented, respectively, by the dashed and dotted lines in Figure 5(a) have a small change, while the results presented, respectively, by the dashed and dotted curves in Figure 5(b) have a large change. Decreasing the slope in case B from 0.3 to 0.29 and 0.28 , respectively, the corresponding results presented, respectively, by the dashed and dotted lines (curves) in Figures 5(a) and 5(b) have a small change. The results presented in Figure 5 show that the minimum in (3) appearing in some special conditions.

\section{Conclusions}

We summarize here our main observations and conclusions.

(a) The rapidity distributions of negatively charged pions produced in $p$ - $p$ collisions and in ${ }^{7} \mathrm{Be}-{ }^{9} \mathrm{Be}$ collisions with different centrality classes at the SPS energies are analyzed by using the revised (three-source) Landau hydrodynamic model. The model results are in agreement with the experimental data measured by the NA61/SHINE Collaboration over an energy range from 6.3 to $17.3 \mathrm{GeV}$. 


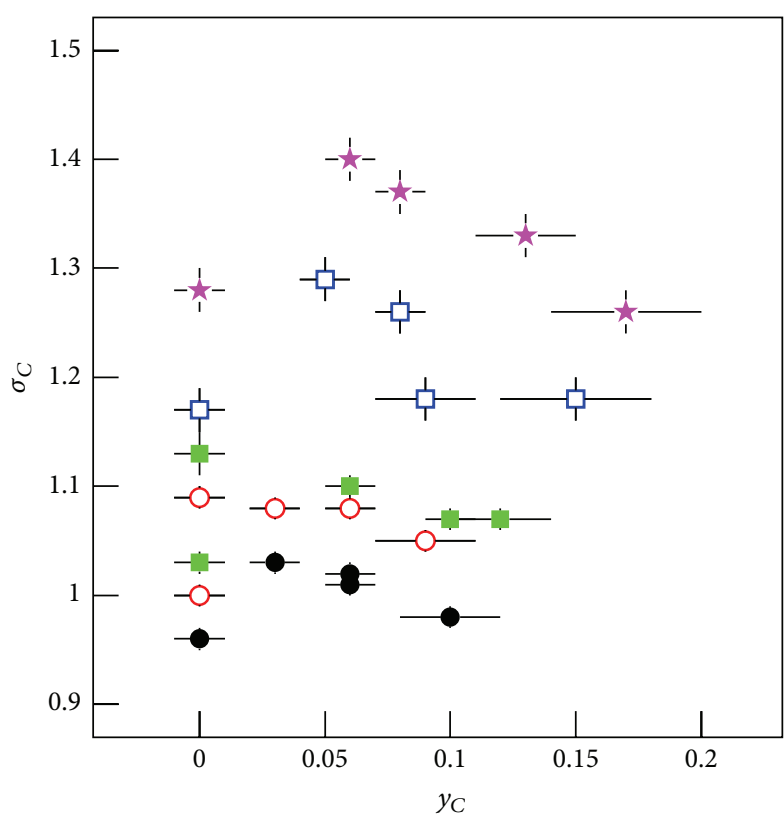

(a)

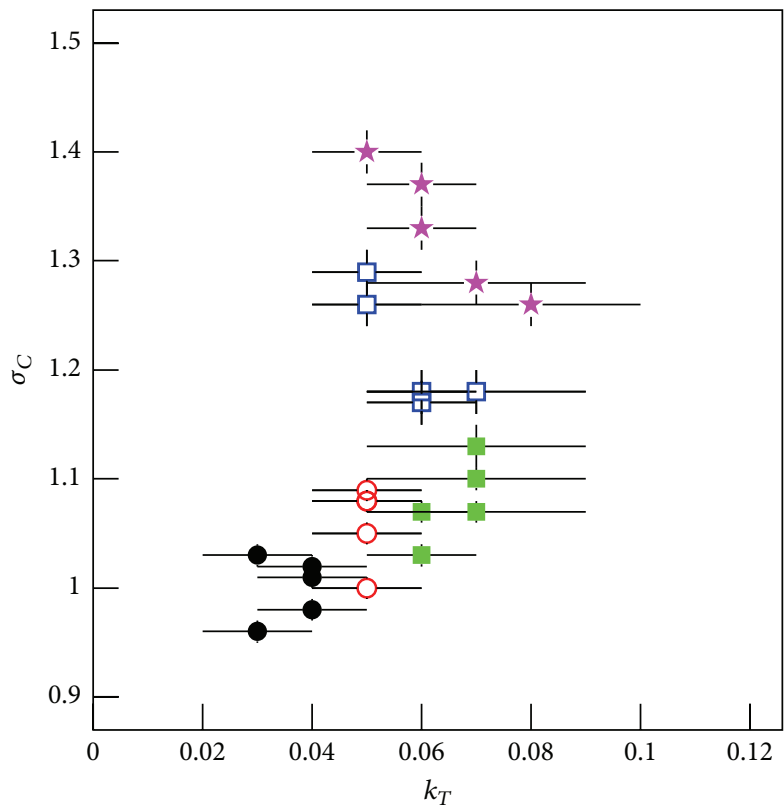

(c)

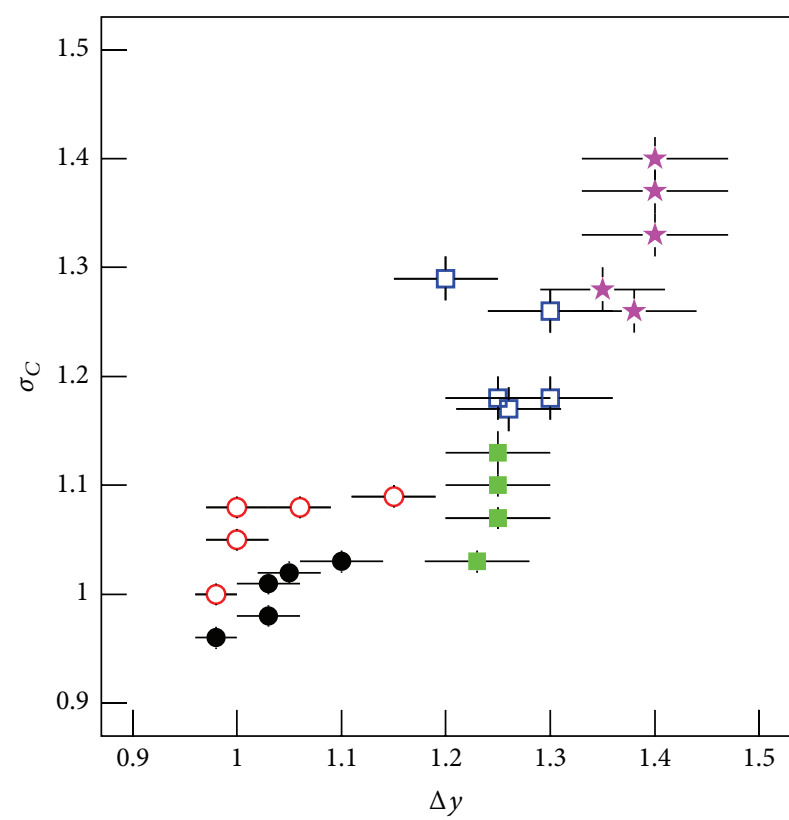

(b)

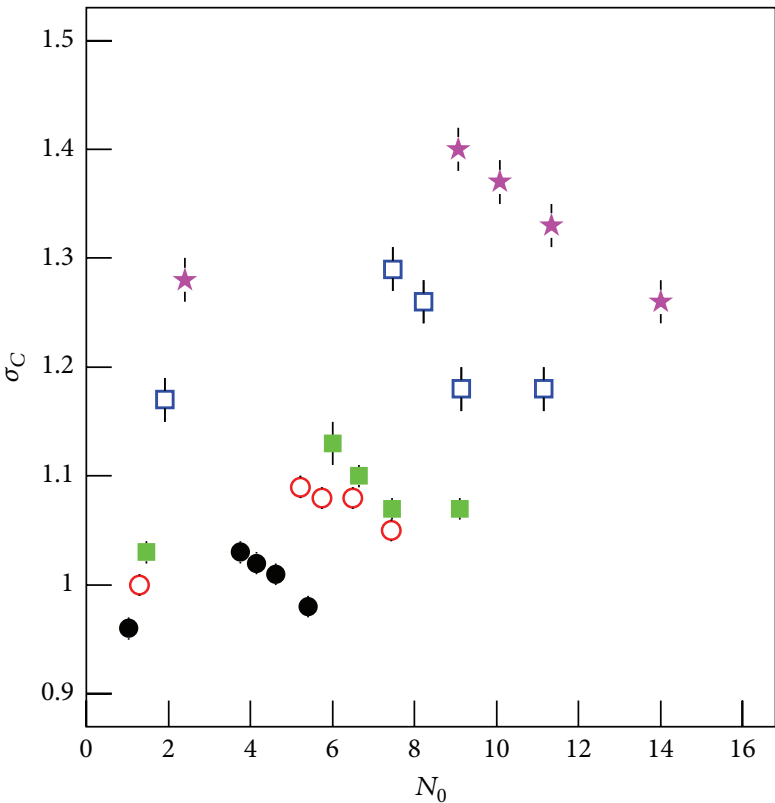

(d)

Figure 4: Dependences of $\sigma_{C}$ on (a) $y_{C}$, (b) $\Delta y$, (c) $k_{T}$, and (d) $N_{0}$. The symbols represent the values listed in Table 1, where the closed circles, open circles, closed squares, open squares, and stars correspond to the collision energy being 6.3, 7.7, 8.8, 12.3 (11.9), and 17.3 (16.8) GeV, respectively.

(b) The values of squared speed-of-sound parameter are extracted from the widths of rapidity distributions. For the target (projectile) source, $c_{s}^{2}(T)\left[c_{s}^{2}(P)\right]$ decreases obviously with increase of $\sqrt{s_{N N}}$ in the considered energy range, and $\sigma_{T}\left(\sigma_{P}\right)$ does not depend on $\sqrt{s_{N N}}$. For the central source, $c_{s}^{2}(C)$ shows a minimum (the softest point in the EoS) at $8.8 \mathrm{GeV}$ when $\sqrt{s_{N N}}$ increases from 6.3 to $17.3 \mathrm{GeV}$, and $\sigma_{C}$ increases obviously with increase of $\sqrt{s_{N N}}$. (c) Combining with our previous works, one can see that $c_{s}^{2}(C)$ is between $1 / 3$ and $1 / 2$ in most cases at higher energies $(\geq 19.6 \mathrm{GeV})$. From 17.3 to $19.6 \mathrm{GeV}, c_{s}^{2}(C)$ has a jump from $\leq 0.3$ to $1 / 3-1 / 2$. In the energy range from the minimum SPS energy to the maximum LHC energy, $\sigma_{C}$ increases monotonously with increase of $\sqrt{s_{N N}}$, though some fluctuations appear in the excitation function. 


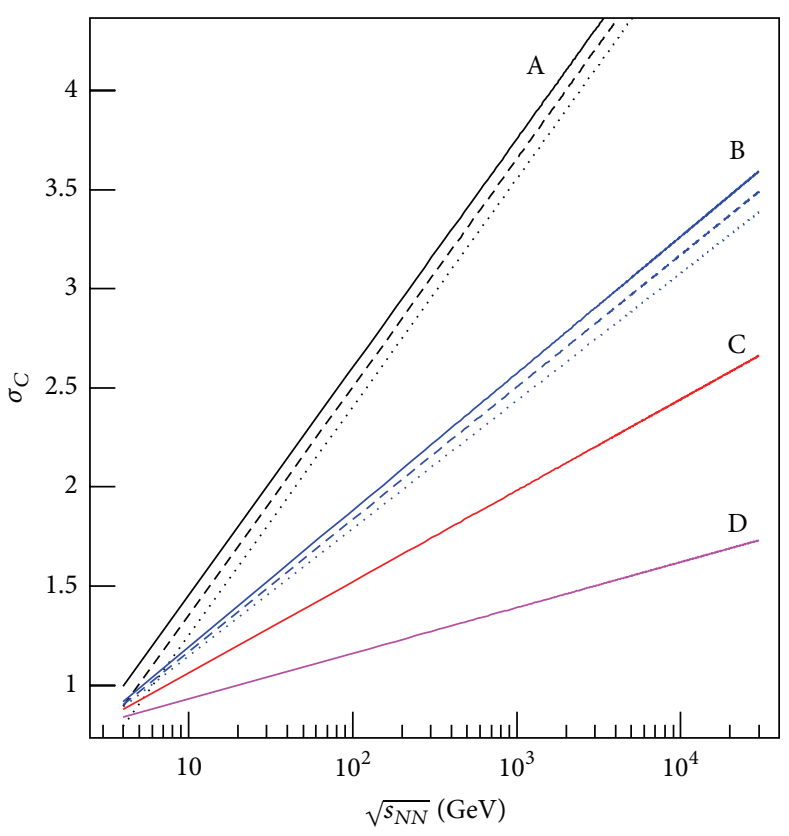

(a)

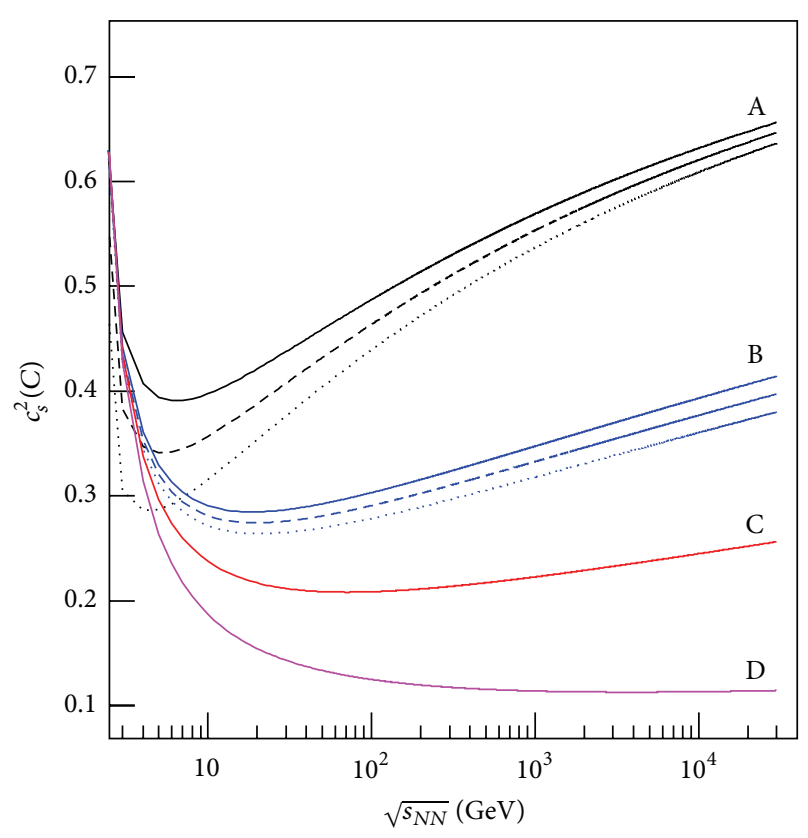

(b)

Figure 5: (a) A few examples of linear relations between $\sigma_{C}$ and $\ln \sqrt{s_{N N}}$. (b) Different linear relations between $\sigma_{C}$ and $\ln \sqrt{s_{N N}}$ corresponding to different dependences of $c_{s}^{2}(C)$ on $\sqrt{s_{N N}}$.

\section{Competing Interests}

The authors declare that there is no conflict of interests regarding the publication of this paper.

\section{Acknowledgments}

The authors would like to thank Dr. Andrzej Wilczek at University of Silesia in Katowice, Poland, and Dr. Emil Aleksander Kaptur at CERN, Switzerland, for supplying us the numerical data of Be-Be collisions in Figure 1 which might be a little bit different from that published in ICPAQGP-2015 [7]. This work was supported by the National Natural Science Foundation of China under Grant no. 11575103 and the US DOE under contract DE-FG02-87ER40331.A008.

\section{References}

[1] J. Adams, M. M. Aggarwal, Z. Ahammed et al., "Multiplicity and pseudorapidity distributions of photons in $\mathrm{Au}+\mathrm{Au}$ Collisions at $\sqrt{s_{N N}}=62.4 \mathrm{GeV}$," Physical Review Letters, vol. 95, Article ID 062301, 2005.

[2] S. S. Adler, S. Afanasiev, C. Aidala et al., "Measurement of density correlations in pseudorapidity via charged particle multiplicity fluctuations in $\mathrm{Au}+\mathrm{Au}$ collisions at $\sqrt{\boldsymbol{s}_{N N}}=200 \mathrm{GeV}$," Physical Review C, vol. 76, Article ID 034903, 2007.

[3] B. B. Back, M. D. Baker, D. S. Barton, R. R. Betts, R. Bindel, and A. Budzanowski, "Charged-particle pseudorapidity density distributions from $\mathrm{Au}+\mathrm{Au}$ Collisions at $\sqrt{s_{N N}}=130 \mathrm{GeV}$," Physical Review Letters, vol. 87, Article ID 102303, 2001.

[4] J. Adam, D. Adamová, M. M. Aggarwal et al., "Pseudorapidity and transverse-momentum distributions of charged particles in proton-proton collisions at $\sqrt{s}=13$ TeV," Physics Letters B, vol. 753, pp. 319-329, 2016.

[5] V. Khachatryan, A. M. Sirunyan, A. Tumasyan et al., "Pseudorapidity distribution of charged hadrons in proton-proton collisions at $\sqrt{s}=13$ Tev," Physics Letters B, vol. 751, pp. 143163, 2015.

[6] N. Abgrall, A. Aduszkiewicz, Y. Ali et al., "Measurement of negatively charged pion spectra in inelastic $\mathrm{p}+\mathrm{p}$ interactions at $p_{\text {lap }}=20,31,40,80$ and $158 \mathrm{GeV} / \mathrm{c}$," European Physical Journal C, vol. 74, article 2794, 2014.

[7] A. Wilczek and E. A. Kaptur, "Recent results from NA61/SHINE on spectra and correlations in $\mathrm{p}+\mathrm{p}$ and $\mathrm{Be}+\mathrm{Be}$ interactions at the CERN SPS," in Proceedings of the 7th International Conference on Physics and Astrophysics of Quark Gluon Plasma (ICPAQGP '15), Kolkata, India, February 2015.

[8] L. D. Landau, "On multiple production of particles during collisions of fast particles," in Collected Papers of L. D. Landau, D. Ter-Haarp, Ed., chapter 74, pp. 569-585, Pergamon Press, Oxford, UK, 1965.

[9] S. Z. Belenkij and L. D. Landau, "A hydrodynamic theory of multiple formation of particles," in Collected Papers of L. D. Landau, D. Ter-Haarp, Ed., chapter 88, pp. 665-700, Pergamon Press, Oxford, UK, 1965.

[10] I. M. Khalatnikov, "Some questions of the relativistic hydrodynamics," Journal of Experimental and Theoretical Physics, vol. 27, pp. 529-541, 1954.

[11] Z. J. Jiang, H. P. Deng, Y. Zhang, and H. Zhang, "Unified descriptions of Hwa-Bjorken and Landau relativistic hydrodynamics and the pseudorapidity distributions in high energy heavy ion collisions," Nuclear Physics Review, vol. 32, pp. 398408, 2015.

[12] P. A. Steinberg, "Bulk dynamics in heavy ion collisions," Nuclear Physics A, vol. 752, pp. 423-432, 2005. 
[13] C.-Y. Wong, "Landau hydrodynamics reexamined," Physical Review C, vol. 78, Article ID 054902, 2008.

[14] E. V. Shuryak, "Multiparticle production in high energy particle collisions," Yadernaya Fizika, vol. 16, pp. 395-405, 1972.

[15] P. Carruthers and M. Duong-Van, "Rapidity and angular distributions of charged secondaries according to the hydrodynamical model of particle production," Physical Review D, vol. 8, pp. 859-874, 1973.

[16] P. Carruthers, "Heretical models of particle production," Annals of the New York Academy of Sciences, vol. 229, pp. 91-123, 1974.

[17] O. V. Zhirov and E. V. Shuryak, "Multiple production of hadrons and predictions of the Landau theory," Yadernaya Fizika, vol. 21, pp. 861-867, 1975.

[18] M. Gazdzicki, M. Gorenstein, and P. Seyboth, "Onset of deconfinement in nucleus-nucleus collisions: review for pedestrians and experts," Acta Physica Polonica B, vol. 42, pp. 307-351, 2011.

[19] S. V. Afanasiev, T. Anticic, D. Barna et al., "Energy dependence of pion and kaon production in central $\mathrm{Pb}+\mathrm{Pb}$ collisions," Physical Review C, vol. 66, Article ID 054902, 2002.

[20] D. T. Larsen, "Energy dependence of hadron spectra and yields in $\mathrm{p}+\mathrm{p}$ and ${ }^{7} \mathrm{Be}+{ }^{9} \mathrm{Be}$ collisions from the NA61/SHINE experiment at the CERN SPS," Journal of Physics: Conference Series, vol. 668, Article ID 012020, 2016.

[21] G. Wolschin, "Pseudorapidity distributions of produced charged hadrons in pp collisions at RHIC and LHC energies," Europhysics Letters, vol. 95, Article ID 61001, 2011.

[22] G. Wolschin, "Relativistic diffusion model," European Physical Journal A, vol. 5, pp. 85-90, 1999.

[23] G. Wolschin, "Diffusion in relativistic systems," Progress in Particle and Nuclear Physics, vol. 59, pp. 374-382, 2007.

[24] G. Wolschin, "Particle production sources at LHC energies," Journal of Physics G: Nuclear and Particle Physics, vol. 40, Article ID 045104, 2013.

[25] L.-N. Gao and F.-H. Liu, "On pseudorapidity distribution and speed of sound in high energy heavy ion collisions based on a new revised Landau hydrodynamic model," Advances in High Energy Physics, vol. 2015, Article ID 184713, 23 pages, 2015.

[26] L.-N. Gao and F.-H. Liu, "On distributions of emission sources and speed-of-sound in proton-proton (proton-antiproton) collisions," Advances in High Energy Physics, vol. 2015, Article ID 641906, 10 pages, 2015.

[27] B. Alver, M. D. Baker, M. Ballintijn et al., "Charged-particle multiplicity and pseudorapidity distributions measured with the PHOBOS detector in $\mathrm{Au}+\mathrm{Au}, \mathrm{Cu}+\mathrm{Cu}, \mathrm{d}+\mathrm{Au}$, and $\mathrm{p}+\mathrm{p}$ collisions at ultrarelativistic energies," Physical Review C, vol. 83, Article ID 024913, 2011.

[28] E. Abbas, B. Abelev, J. Adam et al., "Centrality dependence of the pseudorapidity density distribution for charged particles in $\mathrm{Pb}-\mathrm{Pb}$ collisions at $\sqrt{s_{\mathrm{NN}}}=2.76$ Tev," Physics Letters $B$, vol. 726, pp. 610-622, 2013.

[29] M. Bleicher, "Evidence for the onset of deconfinement from longitudinal momentum distributions? Observation of the softest point of the equation of state," http://arxiv.org/abs/hep-ph/0509314.

[30] J. Steinheimer and M. Bleicher, "Extraction of the sound velocity from rapidity spectra: evidence for QGP formation at FAIR/RHIC-BES energies," European Physical Journal A, vol. 48, article 100, 2012.

[31] L. Kumar and STAR Collaboration, "Identified hadron production from the RHIC beam energy scan," Journal of Physics G: Nuclear and Particle Physics, vol. 38, Article ID 124145, 2011.
[32] A. Rustamov, "The horn, kink and step, dale: from few $\mathrm{GeV}$ to few TeV," Central European Journal of Physics, vol. 10, pp. 12671270, 2012.

[33] Y. B. Ivanov and D. Blaschke, "Robustness of the baryonstopping signal for the onset of deconfinement in relativistic heavy-ion collisions," Physical Review C, vol. 92, no. 2, Article ID 024916, 2015.

[34] Y. B. Ivanov, "Baryon stopping signal for mixed phase formation in HIC," Journal of Physics: Conference Series, vol. 668, Article ID 012061, 2016.

[35] R. A. Lacey, "Indications for a critical end point in the phase diagram for hot and dense nuclear matter," Physical Review Letters, vol. 114, Article ID 142301, 2015.

[36] K.-Y. Kim and I. Zahed, "Baryonic response of dense holographic QCD," Journal of High Energy Physics, vol. 2008, no. 12, article 075, 2008.

[37] E. I. Buchbinder, A. Buchel, S. E. Vázquez et al., "Sound Waves in $(2+1)$ dimensional holographic magnetic fluids," Journal of High Energy Physics, vol. 2008, no. 12, article 90, 2008. 

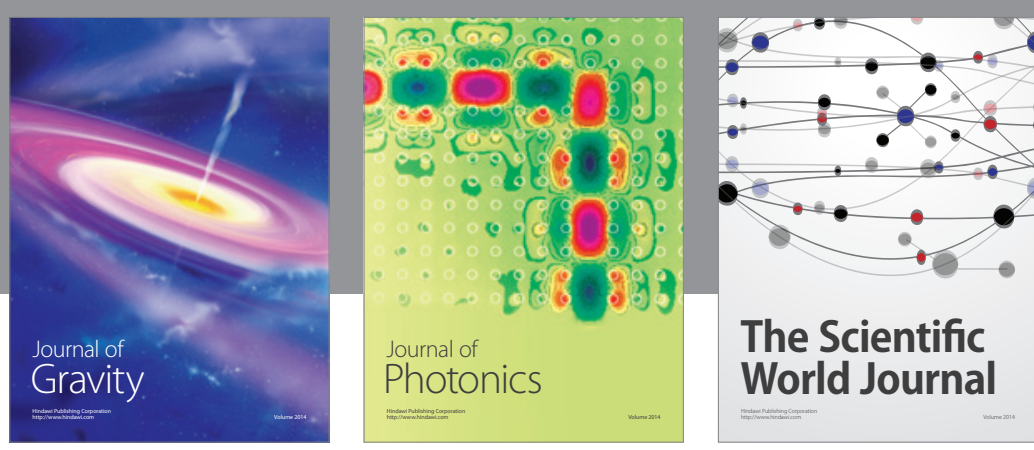

The Scientific World Journal
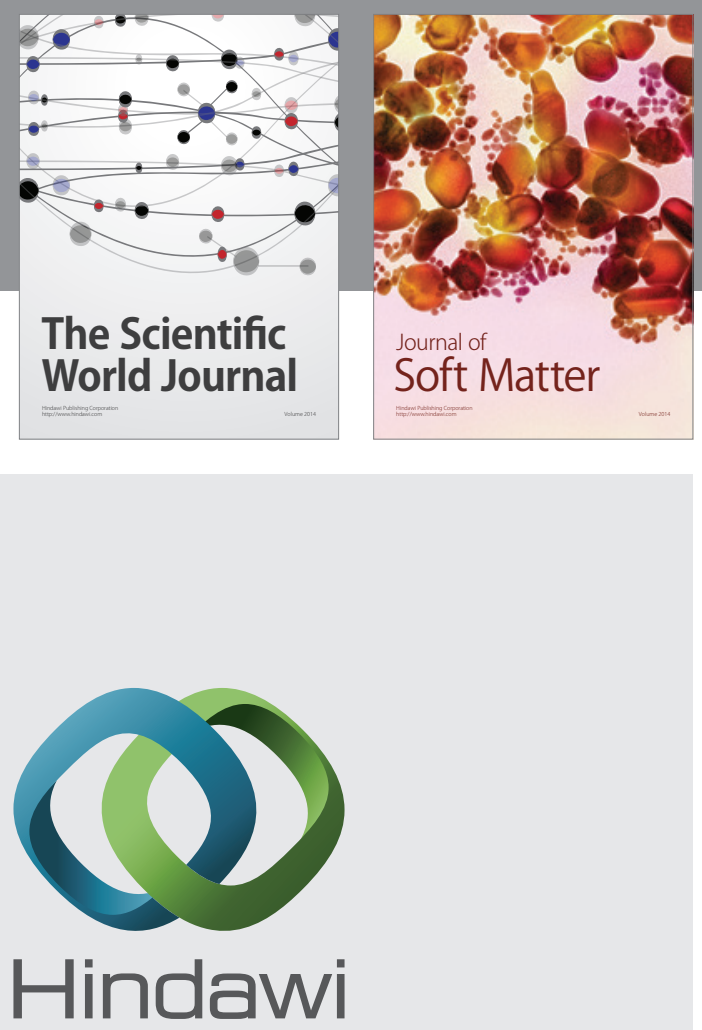

Submit your manuscripts at

http://www.hindawi.com

nternational Journal of

Statistical Mechanics
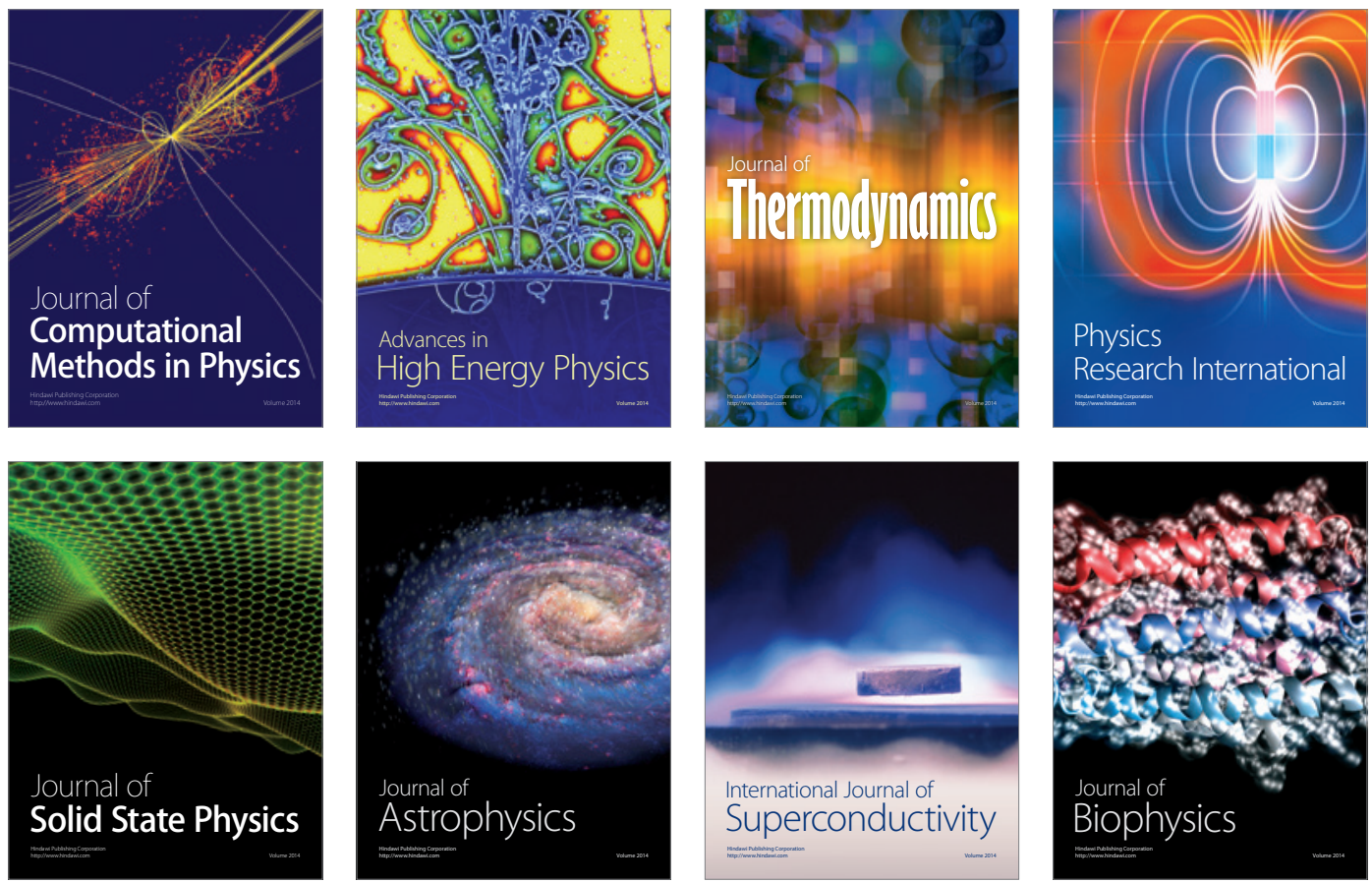
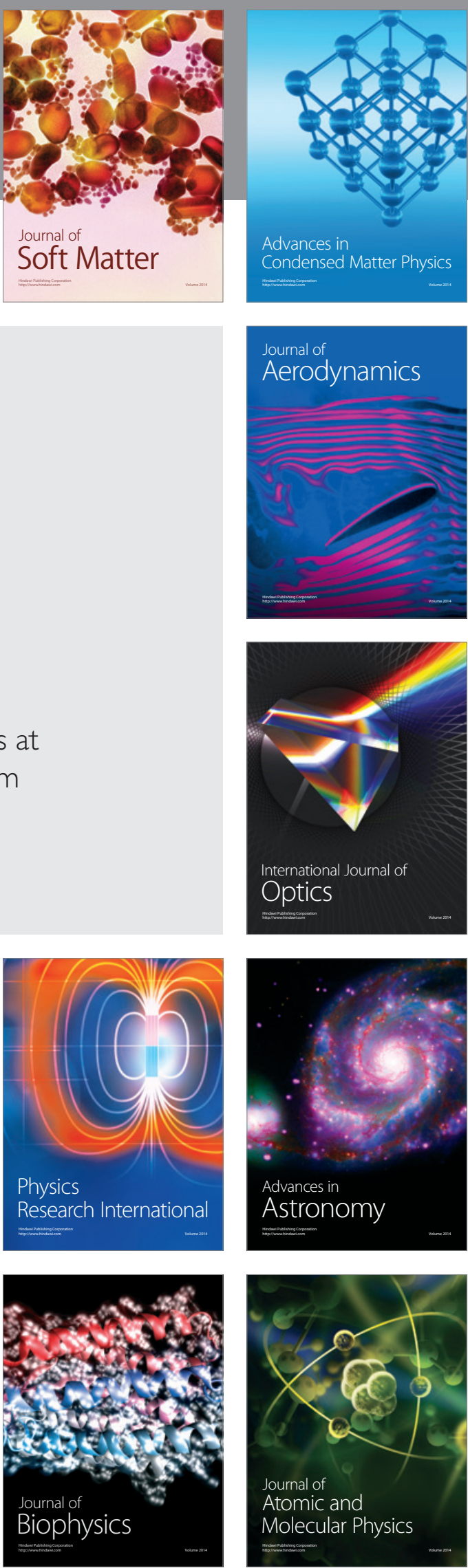